\title{
Target volume dose and clinical outcome in radiotherapy for primary marginal zone lymphoma of the ocular adnexa
}

\author{
NATUHIKO SHIROTA ${ }^{1}$, HIDETSUGU NAKAYAMA ${ }^{1,3}$, SACHICA SHIRAISHI $^{1}$, \\ YOSHIHIKO USUI ${ }^{2}$, KEISUKE KIMURA ${ }^{2}$, TOMOHIDE SANADA ${ }^{1}$, NORIFUMI KENNOKI ${ }^{1}$, \\ MITSURU OKUBO $^{1}$, HIROSHI GOTO ${ }^{2}$ and KOICHI TOKUUYE ${ }^{1}$ \\ Departments of ${ }^{1}$ Radiology and ${ }^{2}$ Ophthalmology, Tokyo Medical University Hospital, Tokyo 160-0023, Japan
}

Received June 27, 2016; Accepted March 17, 2017

DOI: $10.3892 / \mathrm{mco} .2017 .1241$

\begin{abstract}
The aim of the present study was to determine the optimal dose and optimal target volume for marginal zone lymphoma of the ocular adnexa. Between January, 2008 and December, 2013,40 patients with histologically proven primary marginal zone lymphoma of the ocular adnexa who underwent radiotherapy at the Tokyo Medical University were evaluated. The prescribed dose was $30 \mathrm{~Gy}$ in 15 fractions to the iso-center. Doses to the gross tumor volume (GTV), conjunctiva, bulbus oculi, retina and retrobulbar space were investigated, and the association between dose-volume factors and clinical outcome was analyzed. The overall and local progression-free survival rates at 3 and 5 years were $100 \%$ during a median observation period of 32 months. Two patients relapsed in the contralateral ocular adnexa. The progression-free survival rates at 3 and 5 years were 100 and $93.3 \%$, respectively. The mean dose was 2.0 and 1.9 Gy for targets receiving $95 \%$ of the GTV dose (D95). The minimum dose was 0.9-2.0 Gy (median, $1.8 \mathrm{~Gy}$ ). Thus, primary marginal zone lymphoma of the ocular adnexa favorably responded to lower doses of radiation, compared with prescribed doses to the iso-center. A radiation dose of 27 Gy in 15 fractions at D95 of GTV appeared to be optimal.
\end{abstract}

\section{Introduction}

Mucosa-associated lymphoid tissue lymphoma (MALT) was first described by Isaacson et al (1) as a subtype of non-Hodgkin lymphoma and was subsequently classified as marginal zone lymphoma of MALT by the Word Health Organization (2) based on the Revised European-American Classification of lymphoid neoplasms (3). A large national database study reported that marginal zone lymphoma accounted for $\sim 10 \%$

Correspondence to: Dr Hidetsugu Nakayama, ${ }^{3}$ Present address: Department of Radiation Oncology, National Center for Global Health and Medicine, 1-21-1 Toyama, Shinjyuku-ku, Tokyo 162-8655, Japan

E-mail: hnakayama@hosp.ncgm.go.jp

Key words: mucosa-associated lymphoid tissue lymphoma, radiotherapy, ocular adnexa, marginal zone lymphoma of non-Hodgkin lymphomas $(4,5)$ and had a better prognosis compared with diffuse large B-cell lymphoma (4), which has the characteristics of an indolent lymphoma. Extranodal marginal zone lymphoma, which accounted for the majority of marginal zone lymphomas, mainly included the stomach and ocular adnexa. The etiology of marginal zone lymphoma was chronic inflammation, which occurred due to Chlamydia psittaci infection (6).

Non-Hodgkin lymphoma involved the ocular adnexa in $45-75 \%$ of cases of extranodal marginal zone lymphoma, $15-30 \%$ of cases of follicular lymphoma and $10 \%$ of cases of diffuse large B-cell lymphoma (7-11). In ocular adnexal marginal zone lymphoma, the primary sites were the conjunctiva (30-80\%), the retrobulbar soft tissue (10-50\%) and the lacrimal gland (10-55\%) (12-16). Radiotherapy is currently the standard treatment for marginal zone lymphoma of the ocular adnexa. A number of studies have reported that a radiotherapy dose of 24-46 Gy to the entire orbit for primary marginal zone lymphoma of the ocular adnexa was highly effective in terms of local control and survival (12-15). A paucity of reports and the various types of ocular adnexa have made it difficult to undertake an optimal dose volume analysis. Accordingly, a dose volume analysis was conducted herein, based on the ocular adnexa region, to determine the optimal dose-volume association for marginal zone lymphoma of MALT.

\section{Patients and methods}

Patients. This retrospective study was approved by the Ethics Committee of the Tokyo Medical University Hospital (no. 3199). Between January, 2008 and December, 2013, 46 patients underwent radiotherapy for primary non-Hodgkin lymphoma of the ocular adnexa at the Tokyo Medical University Hospital (Tokyo, Japan). A total of 40 patients had marginal zone lymphoma of MALT, 4 had diffuse large B-cell lymphoma, whereas follicular lymphoma and T-cell lymphoma were present in 1 patient each. A total of 40 patients with histologically proven primary marginal zone lymphoma of the ocular adnexa were evaluated. Ophthalmic examination, whole blood count, biochemical examination and body computed tomography (CT) scans were performed prior to radiotherapy initiation. A total of $38(95 \%)$ patients were proven to have immunoglobulin heavy chain rearrangement, 
while the remaining $2(5 \%)$ were not examined. The patient characteristics and radiotherapy method used are summarized in Table I . The median age of the patients was 66.7 years (range, 89.0-26.2 years) and the male/female ratio was 18/22. The most commonly affected sites were the conjunctiva in $19(47.5 \%)$, the retrobulbar soft tissue in $19(47.5 \%)$ and the lacrimal gland in $2(5 \%)$ patients. A total of $33(82.5 \%)$ and 7 $(17.5 \%)$ patients were stage IAE (unilateral) and IIAE (bilateral), respectively, according to the Ann Arbor criteria (17).

All the patients received a radiation dose of $30 \mathrm{~Gy}$ in 15 fractions to the iso-center following immobilization with a custom-made thermoplastic mask. A total of 30 and 10 patients underwent photon and electron therapy, respectively. The 10 (53\%) patients with tumors located in the conjunctiva were irradiated by electrons. The radiotherapy method was individualized based on the involvement site of the ocular adnexa. The clinical target volume (CTV) was determined as the entire orbit, apart from tumors located in the conjunctiva alone. Among the 30 patients irradiated by photons, 19 and 11 patients exhibited primary involvement of the retrobulbar space and conjunctiva, respectively. The number of ports ranged from one to five, which was primarily determined by doctor preference. No patients underwent chemotherapy or antibiotic therapy.

When reviewing the dosimetry, the association between dose volume and clinical outcome was analyzed in 30 patients based on the date treated by three-dimensional (3D) radiotherapy. Dosimetry was analyzed using a treatment planning system (Xio v. 4.6; Elekta AB, Stockholm, Sweden). The growth of the gross tumor volume (GTV) was measured based on the extent of involvement of lymphoma on the CT image, with reference to information from the ophthalmic examination and/or magnetic resonance imaging. Doses to the organs at risk, the conjunctiva, bulbus oculi, retina and retrobulbar space were also estimated. The retrobulbar space was divided into anterior and posterior spaces to evaluate differences in the irradiation doses. When lymphoma was detected bilaterally, both sides of the GTV and the organs at risk were delineated.

Statistical analysis. The overall survival time was calculated from the first day of radiotherapy to the date of death from any cause. The progression-free survival time was calculated from the first day of radiotherapy to the date of the first relapse or the date of death. The time to cataract surgery was calculated from the first day of radiotherapy to the date of the cataract surgery. Continuous variables were analyzed by the Wilcoxon rank-sum test. Paired variables were analyzed by the Wilcoxon signed rank-sum test. Survival time was calculated using the Kaplan-Meier method and the difference was compared using the log-rank test. Stata v.12 software (Stata Co., College Station, TX, USA) was used as the statistical software package. A P-value of $<0.05$ was considered to indicate statistically significant differences. The adverse events were assessed by the Common Terminology Criteria for Adverse Events, version 4.0 (https://www.eortc.be/services/doc/ctc/CTCAE_4.03_2010-06-14_ QuickReference_5x7.pdf).

\section{Results}

Patient survival. No patients experienced local relapse and there were no reported deaths. Thus, the overall survival and
Table I. Patient characteristics and treatment methods $(n=40)$.

Characteristics N

$\begin{array}{lc}\text { Median age, years (range) } & 66.7(26.2-89.0) \\ \text { Gender } & \\ \text { Male:female } & 18: 22\end{array}$

Main involvement site

Conjunctiva:retrobulbar soft

$19: 19: 2$

tissue:lacrimal gland

Laterality

Unilateral:bilateral

Pathology test

Proven:unproven

Gene rearrangement

Positive:negative

Treatment methods

Radiotherapy

Photon:electron

Number of ports

$1: 2: 3: 4: 5$

Photon energy

4MV:6MV

Number of photon ports $(\mathrm{n}=30)$

1:2:3:4:5

1:12:8:8:1

local progression-free survival rates at 3 and 5 years were both $100 \%$ during a median observation period of 32 months (Fig. 1A). Two patients developed recurrence at the contralateral ocular adnexa at 40 and 89 months after radiotherapy. Accordingly, the disease progression-free survival rates at 3 and 5 years were 100 and $93.3 \%$ (95\% confidence interval: 61.3-99\%), respectively. All the patients experienced faint erythema or dry desquamation, which did not progress. Of the 40 patients, 7 (18\%) had already undergone cataract surgery prior to radiotherapy, while $10(30 \%)$ of 33 underwent cataract surgery at a median time of 52 months after radiotherapy (Fig. 1B). The time to cataract surgery did not differ significantly between electron therapy and photon therapy $(\mathrm{P}=0.22)$.

The typical dosimetry for lymphoma involving the primary conjunctiva and the retrobulbar space are shown in Fig. 2A and B. The estimated dose differences to the GTV are shown in Fig. 3A. The mean dose and 95\% received dose (D95) to the GTV were 2.0 and $1.9 \mathrm{~Gy}$, respectively. The minimum dose for the GTV ranged from 0.9 to $2.0 \mathrm{~Gy}$ (median, $1.8 \mathrm{~Gy}$ ). The D95 to the GTV was $\leq 1.8 \mathrm{~Gy}$ in $25 \%$ of the patients. The dose to the GTV compared with the dose to the other orbital sites is shown in Table II. The mean dose and D95 to the conjunctiva were statistically significantly lower compared with those administered to the GTV in conjunctival lymphoma $(\mathrm{P}<0.05)$. The mean dose and D95 to the retrobulbar space was statistically significantly lower compared with those to the GTV in cases of retrobulbar lymphoma $(\mathrm{P}<0.05)$. The mean dose and D95 to the retina were 2.0 Gy (range, 1.8-2.1 Gy) and 2.0 (range, 1.9-2.1 Gy), respectively. 
Table II. GTV dose comparison for the primary site of involvement across various orbital sites.

\begin{tabular}{|c|c|c|c|c|c|c|c|}
\hline \multirow{2}{*}{$\begin{array}{l}\text { Primary involvement } \\
\text { site of lymphoma }\end{array}$} & \multirow{2}{*}{$\begin{array}{c}\text { GTV, } \\
\text { median (range) }\end{array}$} & \multicolumn{2}{|c|}{ Conjunctiva } & \multicolumn{2}{|c|}{ Bulbus oculi } & \multicolumn{2}{|c|}{ Retrobulbar space } \\
\hline & & Median (range) & P-value & Median (range) & P-value & Median (range) & P-value \\
\hline \multicolumn{8}{|l|}{ Mean dose (cGy) } \\
\hline Conjunctivaa $(\mathrm{n}=11)$ & $\begin{array}{c}199 \\
(180-204)\end{array}$ & $\begin{array}{c}190 \\
(150-205)\end{array}$ & $<0.05$ & $\begin{array}{c}202 \\
(196-207)\end{array}$ & N.S. & $\begin{array}{c}198 \\
(149-200)\end{array}$ & N.S. \\
\hline Retrobulbar $(n=19)$ & $\begin{array}{c}200 \\
(171-210)\end{array}$ & $\begin{array}{c}191 \\
(142-212)\end{array}$ & $<0.001$ & $\begin{array}{c}205 \\
(192-214)\end{array}$ & $<0.05$ & $\begin{array}{c}196 \\
(175-203)\end{array}$ & $<0.05$ \\
\hline Total $(\mathrm{n}=30)$ & $\begin{array}{c}200 \\
(171210)\end{array}$ & $\begin{array}{c}191 \\
(142-212)\end{array}$ & $<0.001$ & $\begin{array}{c}204 \\
(192-214)\end{array}$ & $<0.05$ & $\begin{array}{c}197 \\
(149-203)\end{array}$ & $<0.001$ \\
\hline \multicolumn{8}{|l|}{ D95 (cGy) } \\
\hline Conjunctivaa $(\mathrm{n}=11)$ & $\begin{array}{c}189 \\
(155-201)\end{array}$ & $\begin{array}{c}155 \\
(116-202)\end{array}$ & $<0.05$ & $\begin{array}{c}191 \\
(182-201)\end{array}$ & N.S. & $\begin{array}{c}190 \\
(98-195)\end{array}$ & N.S. \\
\hline Retrobulbar $(\mathrm{n}=19)$ & $\begin{array}{c}190 \\
(127-203)\end{array}$ & $\begin{array}{c}171 \\
(109-207)\end{array}$ & $<0.001$ & $\begin{array}{c}195 \\
(148-208)\end{array}$ & N.S. & $\begin{array}{c}185 \\
(127-197)\end{array}$ & N.S. \\
\hline Total $(n=30)$ & $\begin{array}{c}190 \\
(127-203)\end{array}$ & $\begin{array}{c}167 \\
(109-207)\end{array}$ & $<0.001$ & $\begin{array}{c}195 \\
(148-208)\end{array}$ & N.S. & $\begin{array}{c}187 \\
(98-197)\end{array}$ & N.S. \\
\hline
\end{tabular}

ancludes 2 patients with lacrimal gland involvement. GTV, gross tumor volume; N.S., not significant.
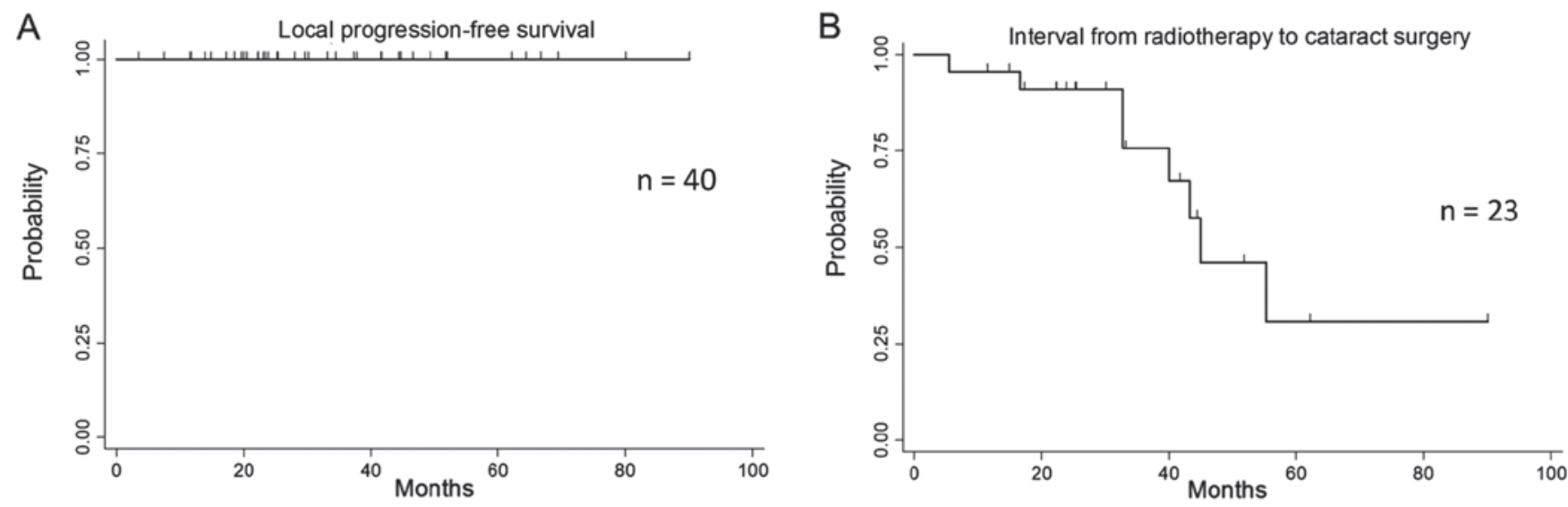

Figure 1. (A) Local progression-free survival curve, estimated by the Kaplan-Meier method. All patients attained complete local control. Two patients, who suffered contralateral ocular adnexal relapse, were successfully treated with radiotherapy. (B) Interval from radiotherapy to cataract surgery, estimated by the Kaplan-Meier method. Of the 40 patients, 7 had already undergone cataract surgery prior to radiotherapy. The time to cataract surgery did not differ significantly between patients treated with electron therapy and those treated with photon therapy $(\mathrm{P}=0.22)$.

An estimated dose comparison between the anterior and posterior retrobulbar spaces is shown in Fig. 2C. The mean dose and D95 to the posterior retrobulbar space (1.9 and $1.8 \mathrm{~Gy}$, respectively) were statistically significantly lower compared with those to the anterior retrobulbar space (2.0 and 1.9 Gy, respectively, $\mathrm{P}<0.001$; Fig. 3B). When restricted only to retrobulbar lymphoma, the mean dose $(\mathrm{P}<0.05)$ and $\mathrm{D} 95$ $(\mathrm{P}<0.001)$ to the posterior retrobulbar space were also statistically significantly lower compared with the anterior space.

\section{Discussion}

Radiotherapy for patients with marginal zone lymphoma of the ocular adnexa achieved good local control with acceptable toxicity (18). Recently, a Japanese study reported that a median dose of 30 Gy in 15 fractions achieved excellent long-term local control for primary marginal zone lymphoma of the ocular adnexa (12). The contralateral orbit is known as the predominant site of distant relapse, although rare $(12,16,18)$. These results indicated that achieving local control was important for patients with primary marginal zone lymphoma of the ocular adnexa and that an investigation of the optimal dose was required.

Currently, the recommended treatment for ocular adnexal lymphoma is to irradiate the entire bony orbit with a dose of 24-25 Gy in 1.5-2.0-Gy fractions, except for cases in which the disease is limited to the conjunctiva (19). This dose is lower than previously published doses $(7,11,13)$. Two studies reported outcomes and a dose analysis for local control of marginal zone lymphoma of ocular adnexa. Fung et al reported that a 

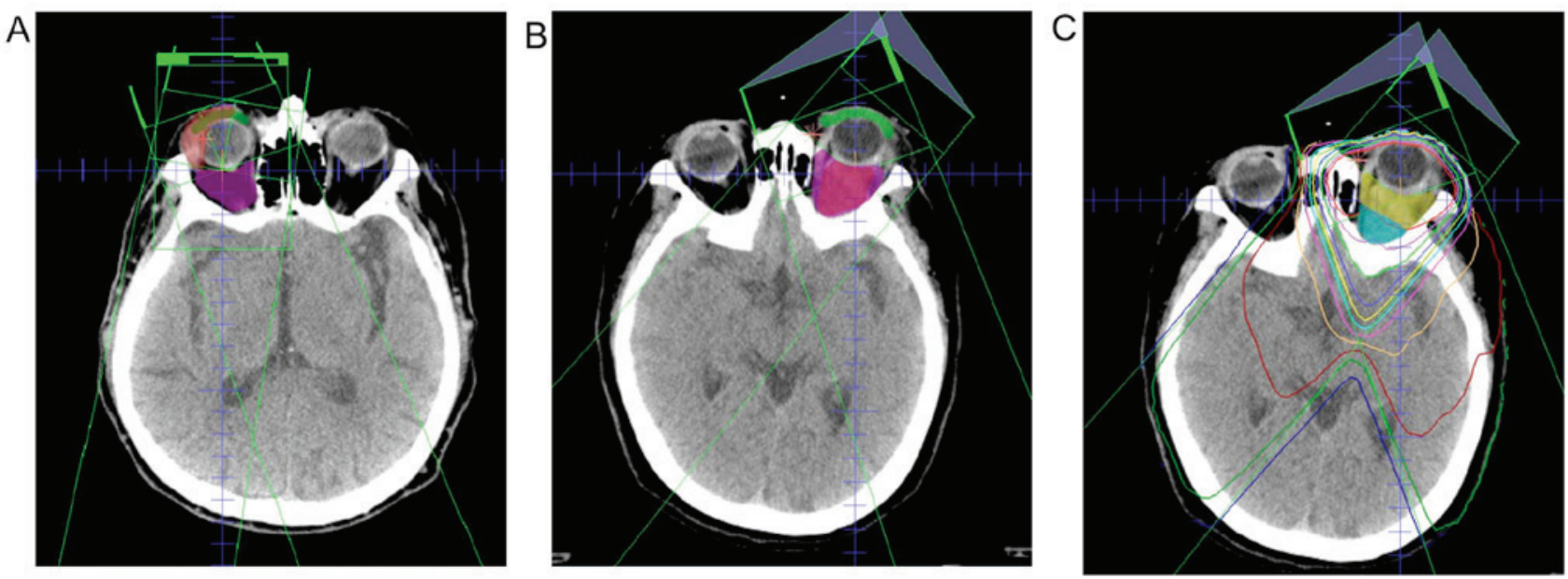

Figure 2. (A) Patients with marginal zone lymphoma of the right conjunctiva received a dose of $30 \mathrm{~Gy}$ in 15 fractions using a non-coplanar technique. (B) Patients with marginal zone lymphoma of the left retrobulbar space received a dose of 30 Gy in 15 fractions using a two-wedge oblique technique. (C) The retrobulbar space was divided into anterior and posterior in reviewing the dosimetry.
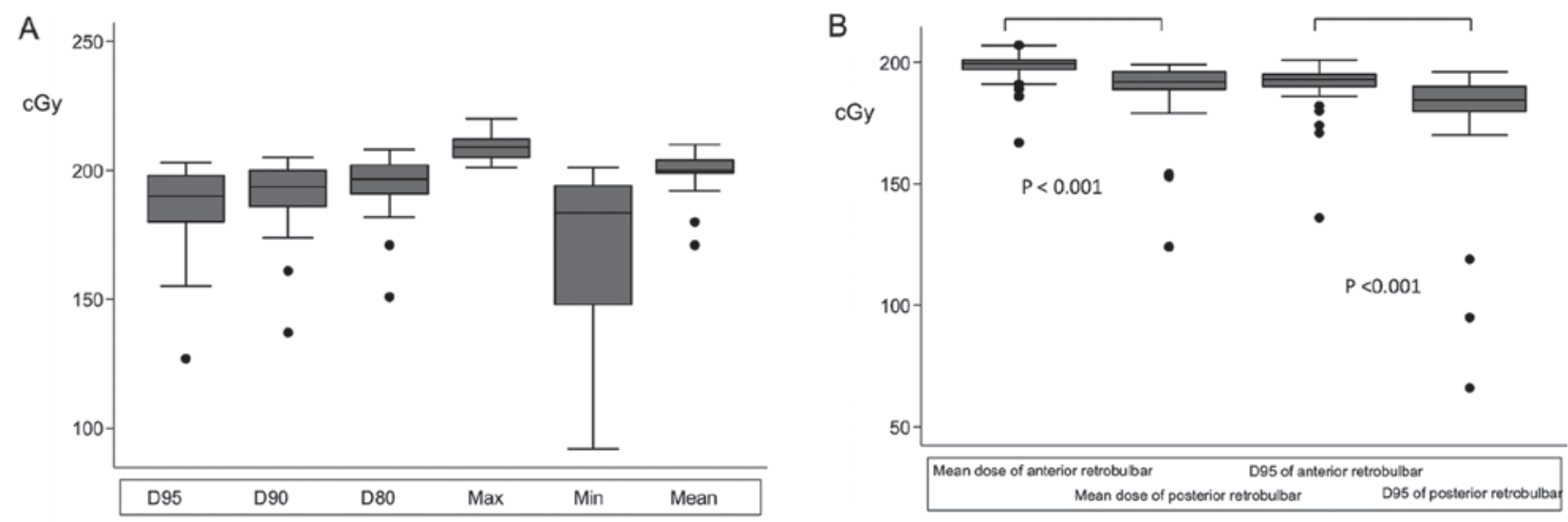

Figure 3. (A) Dose analysis for the gross tumor volume. (B) Dose difference between the anterior and posterior retrobulbar spaces.

dose of $\geq 30$ Gy was superior to a dose of $<30$ Gy in terms of local control for 53 patients with a median observation period of 82 months. Accordingly, they indicated that the optimal dose for ocular adnexal lymphoma was 30.6-32.4 Gy in 1.8 Gy per fraction (7). Bayraktar et al reported that $3 / 18$ patients who received a dose of $<30.6$ Gy relapsed, while $3 / 58$ patients who received a dose of 30.6 Gy relapsed during a median observation period of 5 years. As the former group had a significantly higher relapse rate, they recommended a dose of at least 30.6 Gy for ocular adnexal lymphoma (14).

In our study, all patients were irradiated at a dose of $30 \mathrm{~Gy}$ in 2.0 per fraction at the iso-center, and all patients attained local control. However, $25 \%$ of the patients were irradiated at $1.8 \mathrm{~Gy}$ or lower in the GTV, and doses to the posterior retrobulbar space were $10 \%$ lower compared with the anterior space. These results suggested that a dose of $27 \mathrm{~Gy}$ in $1.8 \mathrm{~Gy}$ per fraction provided favorable local control. Goda et al reported that a radiation dose of $25 \mathrm{~Gy}$ in $2.5 \mathrm{~Gy}$ per fraction achieved $97 \%$ local control after 7 years (13). A dose of $25 \mathrm{~Gy}$ in 2.5 Gy per fraction was found to be equivalent to a dose of $27 \mathrm{~Gy}$ in $1.8 \mathrm{~Gy}$ per fraction, calculated by the linear quadrant model (20).

Several studies have been performed to evaluate low doses for marginal zone lymphoma of the ocular adnexa. Tran et al reported that 24 tumors receiving a dose of 24-25 Gy in 1.5-1.8 Gy per fraction were controlled during a median observation period of 41 months, except for 1 patient who had a local relapse due to a marginal miss (15); they estimated that the irradiated dose in the relapse area was 5-23 Gy.

Regarding low-dose radiotherapy of 4 Gy in 2 fractions, both follicular and marginal zone lymphoma have been studied. Fasola et al reported that 20 patients with a total of 27 sites of ocular adnexal lymphoma were treated with a radiation dose of $4 \mathrm{~Gy}$ in 2 fractions (21). The complete response rate was $85 \%$ during a median observation period of 26 months. Patient histology showed follicular lymphoma in 55\% and marginal zone lymphoma in $40 \%$ of the patients. This response rate was lower compared with that observed in the present study, although the majority had follicular lymphoma. Hoskin et al reported a randomized non-inferiority study comparing a dose of 4 Gy in 2 fractions with 24 Gy in 12 fractions (22). Of the 614 sites treated in 548 patients, $86 \%$ were follicular lymphomas and $14 \%$ were marginal zone lymphomas. A dose of $4 \mathrm{~Gy}$ in 2 fractions did not achieve non-inferiority, compared with a dose of 24 Gy in 12 fractions. Local progression was observed in two groups: One which was administered a dose of 24 Gy to 21 sites, and a second which was administered 
4 Gy to 70 sites. The results of Fasola et al compared favorably with those of Hoskin et al, although the latter study included more cases of follicular lymphoma. Fasola et al treated ocular adnexal lymphoma via 3D conformal radiotherapy, except for cases involving conjunctival tumors. In the study of Hoskin et al, a proportion of the patients were treated by anteroposterior-posteroanterior photon fields.

In Peffer et al, partial orbit treatment was compared with entire orbit treatment in 23 patients (23). A total of $33 \%$ of patients who underwent partial orbit radiotherapy had a relapse in previously uninvolved areas not included in the initial target volume. By contrast, no patients who underwent entire orbit treatment experienced a relapse. Accordingly, it was asserted that irradiation encompassing the entire orbit was necessary and that 25 Gy was effective for local control. As it was difficult to identify the extension of lymphoma to the retrobulbar area, a CTV encompassing the entire orbit is considered to be appropriate.

Kaushik et al reported a risk of retinopathy in 67 patients who received a radiotherapy dose of 18.9-54.0 Gy (24). Eight patients who were irradiated at a dose of 24-40 Gy developed retinopathy at a median of 27 months. The daily fractionated dose of $\geq 2.0$ Gy was a significant risk factor for retinopathy, compared to a dose of $1.8 \mathrm{~Gy}$. A total dose of $30 \mathrm{~Gy}$ or more was not a significant risk, compared to $<30 \mathrm{~Gy}$. As the retina was unavoidable in several cases, a daily dose of 1.8 Gy may be favorable in preventing the onset of retinopathy.

In the present study, no difference in outcome was observed between the conjunctiva and the retrobulbar space as the primary location site. Similarly, Harada et al also reported the absence of prognostic differences between the conjunctiva and other anatomical subsites (12). The same dose was adequate among ocular adnexal anatomical subsites. Goda et al reported that the lacrimal gland and retrobulbar space were associated with high distant relapse rates compared with the conjunctiva (13). The distant relapse rates in Asian studies $(12,16)$, including ours, were relatively lower compared with those reported in Western studies $(7,13)$. The relapse rates in lacrimal gland lymphoma in Western studies $(7,13)$ were higher compared with Asian studies $(12,16)$, and our study may elucidate the reason for this difference. Attention must be focused on the possibility of a distant relapse in lacrimal gland lymphoma.

It was previously demonstrated that electron radiotherapy provides favorable local control $(7,11,13)$ and reduces cataract formation (12) when the lymphoma is located only in the conjunctiva. The association between dose and cataract has been difficult to elucidate, as several patients had cataract due to advanced age. In the present study, the primary endpoint for cataract formation was set to the date of cataract surgery. Electron therapy was more likely to prolong the interval to cataract surgery compared with photon therapy, but the difference was not statistically significant.

The use of an antibiotic therapy targeting Chlamydia psittaci in marginal zone lymphoma of the ocular adnexa has been attempted. According to the majority of the studies, complete response was obtained in only $20 \%$ of the cases (25). From the data, radiotherapy remains the standard treatment, and the addition of antibiotics during radiotherapy may reduce the necessary dose of radiotherapy.
Based on these findings, the optimal dose for the D95 to the GTV currently appears to be 27 Gy in 15 fractions when the lymphoma is located in the retrobulbar space. An entire orbit CTV is most effectively irradiated at a D95 of 25 Gy in 15 fractions. When the lymphoma is limited to the conjunctiva, the GTV is most effectively irradiated at the same dose using electron therapy with a contact lens, whereas irradiation of the retrobulbar space is omitted.

\section{Acknowledgements}

The authors would like to thank RT Kazunari Nakada for his help in retrieving radiation dose volume data.

\section{References}

1. Isaacson P and Wright DH: Malignant lymphoma of mucosa-associated lymphoid tissue. A distinctive type of B-cell lymphoma. Cancer 52: 1410-1416, 1983.

2. Jaffe ES, Harris NL, Stein H, et al: World Health Organization Classification of Tumors: Pathology and Genetics of Tumorus of Haematopoietic and Lymphoid Tissues. IARC Press, Lyon, 2001.

3. Harris NL, Jaffe ES, Stein H, Banks PM, Chan JK, Cleary ML, Delsol G, De Wolf-Peeters C, Falini B, Gatter KC, et al: A revised European-American classification of lymphoid neoplasms: A proposal from the International Lymphoma study group. Blood 84: 1361-1392, 1994.

4. Al-Hamadani M, Habermann TM, Cerhan JR, Macon WR, Maurer MJ and Go RS: Non-Hodgkin lymphoma subtype distribution, geodemographic patterns, and survival in the US: A longitudinal analysis of the National Cancer Data Base from 1998-2011. Am J Hematol 90: 790-795, 2015.

5. Smith A, Howell D, Patmore R, Jack A and Roman E: Incidence of haematological malignancy by sub-type: A report from the Haematological Malignancy research network. Br J Cancer 105: 1684-1692, 2011.

6. Ferreri AJ, Guidoboni M, Ponzoni M, De Conciliis C, Dell'Oro S, Fleischhauer K, Caggiari L, Lettini AA, Dal Cin E, Ieri R, et al: Evidence for an association between Chlamydia psittaci and ocular adnexal lymphomas. J Natl Cancer Inst 96: 586-594, 2004.

7. Fung CY, Tarbell NJ, Lucarelli MJ, Goldberg SI, Linggood RM, Harris NL and Ferry JA: Ocular adnexal lymphoma: Clinical behavior of distinct World Health Organization classification subtypes. Int J Radiat Oncol Biol Phys 57: 1382-1391, 2003.

8. Graue GF, Finger PT, Maher E, Della Rocca D, Della Rocca R, Lelli GJ Jr and Milman T: Ocular adnexal lymphoma staging and treatment: American joint committee on cancer versus Ann Arbor. Eur J Ophthalmol 23: 344-355, 2013.

9. Jenkins C, Rose GE, Bunce C, Wright JE, Cree IA, Plowman N, Lightman S, Moseley I and Norton A: Histological features of ocular adnexal lymphoma (REAL classification) and their association with patient morbidity and survival. Br J Ophthalmol 84: 907-913, 2000.

10. Parikh RR, Moskowitz BK, Maher E, Della Rocca D, Della Rocca R, Culliney B, Shapira I, Grossbard ML, Harrison LB and $\mathrm{Hu} \mathrm{K}$ : Long-term outcomes and patterns of failure in orbital lymphoma treated with primary radiotherapy. Leuk Lymphoma 56: 1266-1270, 2015.

11. Zhou P, Ng AK, Silver B, Li S, Hua L and Mauch PM: Radiation therapy for orbital lymphoma. Int J Radiat Oncol Biol Phys 63: 866-871, 2005.

12. Harada K, Murakami N, Kitaguchi M, Sekii S, Takahashi K, Yoshio K, Inaba K, Morota M, Ito Y, Sumi M, et al: Localized ocular adnexal mucosa-associated lymphoid tissue lymphoma treated with radiation therapy: A long-term outcome in 86 patients with 104 treated eyes. Int J Radiat Oncol Biol Phys 88: 650-654, 2014.

13. Goda JS, Le LW, Lapperriere NJ, Millar BA, Payne D, Gospodarowicz MK, Wells W, Hodgson DC, Sun A, Simpson R and Tsang RW: Localized orbital mucosa-associated lymphoma tissue lymphoma managed with primary radiation therapy: Efficacy and toxicity. Int J Radiat Oncol Biol Phys 81: e659-e666, 2011. 
14. Bayraktar S, Bayraktar UD, Stefanovic A and Lossos IS: Primary ocular adnexal mucosa-associated lymphoid tissue lymphoma (MALT): Single institution experience in a large cohort of patients. Br J Haematol 152: 72-80, 2011.

15. Tran KH, Campbell BA, Fua T, MacManus M, Ryan G, Chesson B and Wirth A: Efficacy of low dose radiotherapy for primary orbital marginal zone lymphoma. Leuk Lymphoma 54: 491-496, 2013.

16. Suh CO, Shim SJ, Lee SW, Yang WI, Lee SY and Hahn JS: Orbital marginal zone B-cell lymphoma of MALT: Radiotherapy results and clinical behavior. Int J Radiat Oncol Biol Phys 65: 228-233, 2006.

17. Carbone PP, Kaplan HS, Musshoff K, Smithers DW and Tubiana M: Report of the committee on hodgkin's disease staging classification. Cancer Res 31: 1860-1861, 1971.

18. Stefanovic A and Lossos IS: Extranodal marginal zone lymphoma of the ocular adnexa. Blood 114: 501-510, 2009.

19. Yahalom J, Illidge T, Specht L, Hoppe RT, Li YX, Tsang R and Wirth A; International Lymphoma Radiation Oncology Group: Modern radiation therapy for extranodal lymphomas: Field and dose guidelines from the international lymphoma radiation oncology group. Int J Radiat Oncol Biol Phys 92: 11-31, 2015.

20. Fowler JF: The linear-quadratic formula and progress in fractionated radiotherapy. Br J Radiol 62: 679-694, 1989.
21. Fasola CE, Jones JC, Huang DD, Le QT, Hoppe RT and Donaldson SS: Low-dose radiation therapy (2 Gy x 2) in the treatment of orbital lymphoma. Int J Radiat Oncol Biol Phys 86: 930-935, 2013

22. Hoskin PJ, Kirkwood AA, Popova B, Smith P, Robinson M, Gallop-Evans E, Coltart S, Illidge T, Madhavan K, Brammer C, et al: 4 Gy versus 24 Gy radiotherapy for patients with indolent lymphoma (FORT): A randomised phase 3 non-inferiority trial. Lancet Oncol 15: 457-463, 2014.

23. Pfeffer MR, Rabin T, Tsvang L, Goffman J, Rosen N and Symon Z: Orbital lymphoma: Is it necessary to treat the entire orbit? Int J Radiat Oncol Biol Phys 60: 527-530, 2004.

24. Kaushik M, Pulido JS, Schild SE and Stafford S: Risk of radiation retinopathy in patients with orbital and ocular lymphoma. Int J Radiat Oncol Biol Phys 84: 1145-1150, 2012.

25. Kiesewetter B and Raderer M: Antibiotic therapy in nongastrointestinal MALT lymphoma: A review of the literature. Blood 122: 1350-1357, 2013. 